\title{
CORRECTION
}

\section{Correction to: The catecholaldehyde hypothesis: where MAO fits in}

\author{
David S. Goldstein ${ }^{1}$
}

Published online: 21 December 2019

(c) This is a U.S. government work and not under copyright protection in the U.S.; foreign copyright protection may apply 2019

\section{Correction to: Journal of Neural Transmission https://doi.org/10.1007/s00702-019-02106-9}

The original version of this article unfortunately contained a mistake. The presentation of Fig. 1 was incorrect. The corrected Fig. 1 is given below.

The original article has been corrected.
The original article can be found online at https://doi.org/10.1007/ s00702-019-02106-9.

David S. Goldstein

goldsteind@ninds.nih.gov

1 Autonomic Medicine Section, Clinical Neurosciences Program, Division of Intramural Research, National Institute of Neurological, Disorders and Stroke, National Institutes of Health, 9000 Rockville Pike MSC-1620, Building 10 Room 8N260, Bethesda, MD 20892-1620, USA

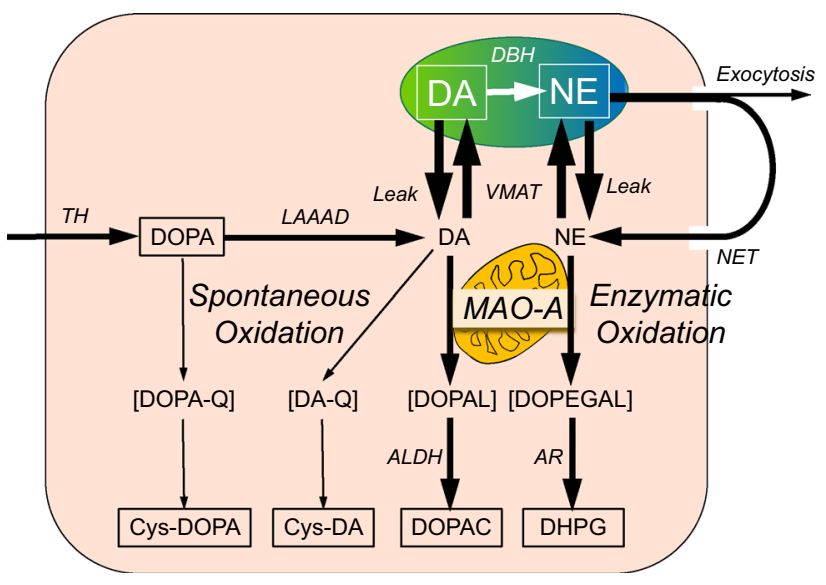

Fig. 1 Overview of the sources and fate of intra-neuronal catecholamines, with emphasis on enzymatic oxidation catalyzed by MAO. Dopamine (DA) is produced in the cytoplasm via tyrosine hydroxylase $(\mathrm{TH})$ acting on tyrosine to form 3,4-dihydroxyphenylalanine (DOPA) and then L-aromatic-amino-acid decarboxylase (LAAAD) acting on DOPA to form dopamine. Most of cytoplasmic DA is taken up into vesicles by way of the vesicular monoamine transporter (VMAT). Dopamine-beta-hydroxylase (DBH) in the vesicles catalyzes the production of norepinephrine (NE) from DA. Cytoplasmic DA is subject to oxidative deamination catalyzed by monoamine oxidase-A (MAO-A) in the outer mitochondrial membrane to form 3,4-dihydroxyphenylacetaldehyde (DOPAL), and NE is deaminated to form 3,4-dihydroxyphenylglycolaldehyde (DOPEGAL). DOPAL is converted to 3,4-dihydroxyphenylacetic acid (DOPAC) via aldehyde dehydrogenase (ALDH), and DOPEGAL is converted to 3,4-dihydroxyphenylglycol (DHPG) via aldehyde/aldose reductase (AR). Most of vesicular DA and NE released by exocytosis is taken back up into the cytoplasm via cell membrane transporters-the NET for NE (although DA is a better substrate than NE for uptake via the NET). DOPA can undergo spontaneous oxidation to DOPA-quinone (DOPA-Q), resulting in formation of 5-S-cysteinylDOPA (CysDOPA), and DA can undergo spontaneous oxidation to DA-quinone (DA-Q), resulting in formation of 5-S-cysteinylDA (Cys-DA) 